\title{
LINEAR ALGEBRAS*
}

\author{
BY

\section{LEONARD EUGENE DICKSON}

We consider linear algebras of which neither the associative nor the commutative law of multiplication is assumed, but which contain a modulus. It is shown in $\S 2$ that every element $X$ satisfies an equation whose degree is the number of units and which is derived from the well-known characteristic equation of a linear associative algebra by replacing $X^{3}$ by $X(X X)$, etc. In $\S 3$ there is developed a very simple theory of polynomials in these quasi powers $X X$, $X(X X)$, etc.

Linear algebras in which every element satisfies a quadratic equation are treated in $\$ \S 5-7$. By making either of two sets of further assumptions, we obtain algebras of the quaternion type.

While in $\$ \S 1-7$ the coördinates range over the elements of a general field (domain of rationality), we restrict attention in $\S 8$ to commutative linear algebras in the field of real numbers with division always possible and unique. $\dagger$ It is shown that the numbers of units is at least six, and that if there be only six units the characteristic sextic is the equation of lowest degree which is satisfied by the general element of the algebra. Of linear algebras with a modulus, with real coördinates and with division uniquely possible, multiplication is associative but not commutative in the quaternion system, whereas multiplication is commutative but not associative in no algebra with fewer than six units.

In $\S 9$ we exhibit commutative linear algebras with six units in which every element is a root of a quartic, but not of a quadratic or cubic, equation.

In $\S 10$ it is shown $\ddagger$ that division is always possible and unique in CAYLEY's non-associative linear algebras in eight units with real coördinates.

1. Definitions and notations. - Given a field (domain of rationality) $F$ and $n$ elements $e_{1}, \cdots, e_{n}$, linearly independent with respect to $F$, and such that

$$
e_{i} e_{j}=\sum_{k=1}^{n} \gamma_{i j k} e_{k} \quad(i, j=1, \ldots, n ; \gamma \text { 's in } F),
$$

\footnotetext{
* Presented to the Society at Minneapolis, December 28, 1910.

$\dagger$ For an extensive class of fields, not including the field of reals, commutative linear algebras in which division is uniquely possible are given in my papers in these Transactions, vol. 7 (1906), pp. 370-390, 514-522.
}

$\ddagger$ Added November, 1911; presented to the Society at Chicago; December 29, 1911. 
we shall say that the set of elements $\Sigma x_{k} e_{k}$, in which the $x_{k}$ range over $F$, form a linear algebra. The sum, difference and product of two elements $X=\Sigma x_{i} e_{i}$ and $X^{\prime}=\Sigma x_{i}^{\prime} e_{i}$ are defined by

$$
X \pm X^{\prime}=\sum_{i=1}^{n}\left(x_{i} \pm x_{i}^{\prime}\right) e_{i}, \quad X X^{\prime}=\sum_{i, j=1}^{n} x_{i} x_{j}^{\prime} e_{i} e_{j}=\sum_{i, j, k} x_{i} x_{j}^{\prime} \gamma_{i j k} e_{k} .
$$

ultiplication is not assumed to be associative or commutative.

Given two elements $X$ and $Y$ of the algebra, $X \neq 0$, we can determine an e ement $X^{\prime}$ such that $X X^{\prime}=Y$ if

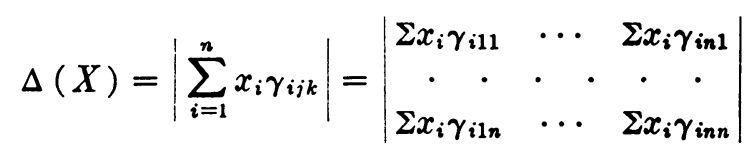

is not zero; we can determine $X^{\prime}$ such that $X^{\prime} X=Y$ if

$$
\Delta^{\prime}(X)=\left|\sum_{i=1}^{n} x_{i} \gamma_{j i k}\right|
$$

ss not zero. We call $\Delta(X)$ the right hand determinant of $X$, and $\Delta^{\prime}(X)$ the left hand determinant of $X$.

We shall assume that $e_{1}$ is a modulus, viz., that $e_{1} X=X e_{1}=X$ for every element $X$ We shall write 1 for $e_{1}$. Then, by (1) for $i=1$ and $j=1$ in turn,

$$
\gamma_{1 j i}=\gamma_{j 1 i}=1, \quad \gamma_{1 j k}=\gamma_{j 1 k}=0
$$

Thus $x_{1}$ occurs only in the terms of $\Delta$ and $\Delta^{\prime}$ lying in the main diagonal. Hence if we replace $x_{1}$ by $x_{1}-\rho$, where $\rho$ is an element of $F$, we obtain

$$
\Delta(X-\rho)=\sum_{i=0}^{n} r_{i} \rho^{i}, \quad \Delta^{\prime}(X-\rho)=\sum_{i=0}^{n} l_{i} \rho^{i},
$$

called the right and left hand characteristic determinants of $X$. Equating them to zero, we obtain the right and left hand characteristic equations.

Avoiding the ambiguous symbol $X^{i}$, we shall set

$X_{0}={ }_{0} X=1, \quad X_{1}=X, \quad X_{i+1}=X_{i} X, \quad{ }_{1} X=X, \quad{ }_{i+1} X=X\left({ }_{i} X\right)$.

If $\Sigma t_{i} X_{i}=0, X$ is called a right hand root of the equation $\Sigma t_{i} \rho^{i}=0$; if $\Sigma_{i}\left({ }_{i} X\right)=0, X$ is a left hand root.

2. Theorem. Every element of a linear algebra is a left hand root of the right and characteristic equation and a right hand root of the left hand characteristic equation; namely, for any element $X$,

$$
\sum_{i=0}^{n} r_{i}\left({ }_{i} X\right)=0, \quad \sum_{i=0}^{n} l_{i} X_{i}=0 .
$$


Denote by $x_{1}^{\prime}, \cdots, x_{n}^{\prime}$ the co-factors of the elements in the first row of the determinant $\Delta(X)$. Multiply them by the corresponding elements of the $k$ th row of $\Delta(X)$ and add the products. We obtain the coefficient $y_{k}$ of $e_{k}$ in $Y=X X^{\prime}$. Hence $y_{1}=\Delta, y_{k}=0(k>1)$. Thus ${ }^{\prime} X X^{\prime}=\Delta$. Replace $x_{1}$ by $x_{1}-\rho$ and let $X^{\prime}$ become $F=\Sigma_{i=0}^{n-1} F_{i} \rho^{i}$, where the $F_{i}$ are elements of the algebra. Then

$$
(X-\rho) F=\sum_{i=0}^{n} r_{i} \rho^{i}
$$

Equating coefficients of like powers of $\rho$, we get

$$
X F_{0}=r_{0}, X F_{1}-F_{0}=r_{1}, \cdots, X F_{n-1}-F_{n-2}=r_{n-1},-F_{n-1}=r_{n} .
$$

Multiply on the left the second equation by $X$, the third by $X$ twice, the fourth by $X$ three times, etc. By adding we get $\Sigma r_{i}\left({ }_{i} X\right)=0$.

The proof* of $\Sigma l_{i} X_{i}=0$ is similar.

3. Linear combinations of the right hand $\dagger$ powers $X_{i}$ of an element $X$ of a linear algebra have certain properties analogous to those of polynomials in a scalar variable. Set

$$
B_{0}=\sum_{j=0}^{\beta} b_{j} X_{j}, \quad B_{k}=B_{k-1} X=\sum_{j=0}^{\beta} b_{j} X_{j+k} .
$$

Lemma. If $a=b c+d$, where

$$
a=\sum_{i=0}^{a} a_{i} \rho^{i}, \quad b=\sum_{i=0}^{\beta} b_{i} \rho^{i}, \quad c=\sum_{i=0}^{\gamma} c_{i} \rho^{i}, \quad d=\sum_{i=\imath}^{\delta} d_{\imath} c^{i},
$$

then, for every element $X$ of a linear algebra,

$$
\sum_{i=0}^{a} a_{i} X_{i}=\sum_{k=0}^{\gamma} c_{k} B_{k}+\sum_{i=0}^{\delta} d_{i} X_{i}
$$

For proof, set $a_{i}^{\prime}=a_{i}-d_{i}, a^{\prime}=a-d$. From $a^{\prime}=b c$, we have

$$
a_{i}^{\prime}=\sum_{k=g}^{s} c_{k} b_{i-k} \quad(g=\text { greater of } 0, i-\beta ; s=\text { smaller of } ;
$$

For $0 \leqq k \leqq \gamma$, the coefficients of $X_{i}$ in $B_{k}$ is $b_{i-k}$ or zero according as $i-\beta \leqq ? \leqq i$ or not.

Corollary I. If $X$ is a root of

$$
B_{0}=\sum_{j=0}^{\beta} b_{i} X_{j}=0
$$

* Bulletin of the American Mathematical Society, vol. 17 (1911), p. 294. Under the assumption that multiplication is associative, the proof becomes essentially that by Frobenrus, Berliner Sitzungsberich t e, 1896, p. 601.

$\dagger$ Throughout the section we may replace right hand by left hand powers. 
and if

is a factor of

$$
\sum_{j=0}^{\beta} b_{j} \rho^{j}
$$

then

$$
\sum_{i=0}^{a} a_{i} \rho^{i}
$$

$$
\sum_{i=0}^{a} a_{i} X_{i}=0
$$

Corollary II. If $X$ is a root of $\Sigma b_{j} X_{j}=0$, but of no similar equation of lower degree, then $\Sigma a_{i} X_{i}=0$ implies that $\Sigma a_{i} \rho^{2}$ has the factor $\Sigma b_{j} \rho^{j}$.

Theorem. If $a=\Sigma a_{i} \rho^{i}$ and $b=\Sigma b_{i} \rho^{i}$ have the greatest common divisor $g=\Sigma g_{i} \rho^{i}$, and we set $G_{0}=\Sigma g_{i} X_{i}, G_{s}=G_{s-1} X$, etc., then

$$
A_{0}=\Sigma m_{s} G_{s}, \quad B_{0}=\Sigma n_{t} G_{t}, \quad G_{0}=\Sigma p_{s} A_{s}+\Sigma q_{t} B_{t},
$$

where the $m, n, p, q$ are elements of $F$. In particular, $A_{0}$ and $B_{0}$ both vanish if and only if $G_{0}$ vanishes.

To simplify the notation, let Euclid's process to determine $g$ terminate in three steps, so that

$$
a=b c+d, \quad b=d e+f, \quad d=f k+g, \quad f=g .
$$

In view of the above Lemma, we have

$$
\begin{array}{ll}
A_{0}=\sum_{k=0}^{\gamma} c_{k} B_{k}+D_{0}, & B_{0}=\sum_{k=0}^{e} e_{k} D_{k}+F_{0}, \\
D_{0}=\sum_{k=0}^{\kappa} k_{k} F_{k}+G_{0}, & F_{0}=\sum_{k=0}^{\lambda} l_{k} G_{k} .
\end{array}
$$

By the last two equations, $D_{0}=\Sigma v_{j} G_{j}$; by the second, $B_{0}=\Sigma n_{t} G_{t}$; by the first, $A_{0}=\Sigma m_{s} G_{s}$. Next, the second equation gives

$$
B_{k}=\sum_{j=0}^{\epsilon} e_{j} D_{j+k}+F_{k}
$$

Replacing $D_{j+k}$ by its value from the first equation, we see that the third equation gives $G_{0}$ as a linear element of the $A_{s}$ and $B_{t}$.

4. Suppose that the general element $X$ is a root of

$$
{ }_{m} X=b_{1}\left({ }_{m-1} X\right)+\cdots+b_{m-1} X+b_{m},
$$

but of no similar equation of lower degree. By the preceding Cor. II, $b_{m}$ is a 
factor of the right hand characteristic determinant $\Delta(X)$. Set

$$
\Delta=b_{m} Q, \quad C={ }_{m-1} X-b_{1}\left({ }_{m-2} X\right)-\cdots-b_{m-1}=\sum_{i=1}^{n} c_{i} e_{i} .
$$

Then $X C=b_{m}$. Let $m_{1}, \cdots, m_{n}$ be the co-factors of the elements of the first row of $\Delta(X)$ and set $M=\Sigma m_{i} e_{i}$. By $\S 2, X M=\Delta$. Since $Q$ is a scalar,

$$
X(Q C)=(X C) Q=b_{m} Q=\Delta=X M, \quad X(M-Q C)=0 .
$$

Then $X X^{\prime}=0$ for every $X$, where $X^{\prime}=M-Q C$. The coördinates $x_{j}^{\prime}$ of $X^{\prime}$ are polynomials in $x_{1}, \cdots, x_{n}$. Then $x_{j}^{\prime} \Delta(x)$ is identically zero in $x_{1}, \cdots, x_{n}$, while $\Delta(x)$ is not $(\S 1)$. Thus $x_{j}^{\prime}=0, X^{\prime}=0$. Hence $M=Q C, m_{i}=Q c_{i}$.

\section{Linear algebras in which every element satisfies a quadratic equation.*}

5 . Let the square of every element be a linear function of that element with coefficients in a field $F$ not having modulus 2 . Since $e^{2}=a+b e,(e-b / 2)^{2}$ is an element of $F$. Hence we take the fundamental units to be $1, e_{1}, \cdots, e_{m}$, where $e_{i}^{2}=s_{i i}$, an element of $F$. We shall introduce new units $1, E_{1}, \cdots, E_{m}$ such that

$$
E_{k}^{2}=c_{k}, \quad E_{k} E_{l}+E_{l} E_{k}=0 \quad(l, k=1, \ldots, m ; l \neq k),
$$

where the $c_{k}$ are elements of $F$. The case $m=1$ requires no attention. For $m>1, i \neq j,\left(e_{i} \pm e_{j}\right)^{2}$ is a linear function of $e_{i} \pm e_{1}$. Hence $e_{i} e_{j}+e_{j} e_{i}$ is a linear function of $e_{i}+e_{j}$, also a linear function of $e_{i}-e_{j}$, and hence is an element of $F$ :

$$
e_{i} e_{j}+e_{j} e_{i}=2 s_{i j}=2 s_{j i} \quad(i, j=1, \ldots, m) .
$$

Let $u_{1}, \cdots, u_{m}$ be arbitrary e'ements of $F$ and set $U=\Sigma u_{k} e_{k}$. Then

$$
U^{2}=Q \equiv \sum_{k, l=1}^{m} s_{k l} u_{k} u_{l}
$$

By a linear transformation with coefficients in $F$,

$$
u_{k}=\sum_{l=1}^{m} a_{k l} v_{l} \quad(k=1, \ldots, l ;|a| \neq 0)
$$

$Q$ can be reduced to the form $\Sigma c_{i} v_{i}{ }^{2}$. Set

$$
E_{l}=\sum_{k=1}^{m} a_{k l} e_{k} \quad(l=1, \ldots, m) .
$$

* Sections 5-7 were read before the Mathematical Club of the University of Chicago, May 18, 1906. 
Then $1, E_{1}, \cdots, E_{m}$ are linearly independent with respect to $F$ and may be taken as new units. Now

$$
U=\sum_{k, l} a_{k l} v_{l} e_{k}=\sum_{l} v_{l} E_{l}, \quad U^{2}=\sum_{k, l} v_{k} v_{l} E_{k} E_{l}=\dot{\Sigma} c_{i} v_{i}^{2} .
$$

Hence relations (4) hold.

Conversely, when these relations hold, every element

$$
X=x_{0}+\sum_{k=1}^{m} x_{k} E_{k}
$$

of the algebra satisfies a quadratic equation:*

$$
X^{2}-2 x_{0} X+\sigma=0, \quad \sigma \equiv x_{0}^{2}-\sum_{k=1}^{m} c_{k} x_{k}^{2} .
$$

This quadratic is the eruation of lowest degree with coefficients in $F$ satisfied by an element $X$ not in $F$. Thus, by $\S 4, \sigma$ is a factor of $\Delta(X)$ and the cofactors of the elements of the first row of $\Delta$ are $q x_{0},-q x_{1}, \cdots,-q x_{m}$, where $q=\Delta / \sigma$.

If $X$ is a root of a quadratic, all products of $n$ factors each $X$ are equal and may be designated by $X^{n}$.

6. Consider algebras (4) for which a product vanishes only when one factor vanishes. Then each $c_{k}$ is a not-square in $F$. Further, $E_{i} E_{j}$ is linearly independent of $1, E_{i}, E_{j}$. For, if

then

$$
E_{i} E_{j}=a+b E_{i}+c E_{j}
$$

$$
\left(E_{i}-c\right)\left(E_{j}+s E_{i}+t\right) \cdot=0, \quad s=\frac{a+b c}{c^{2}-c_{i}}, \quad t=\frac{b c_{i}+a c}{c^{2}-c_{i}}
$$

Hence $m \geqq 3$ and, by interchanging $E_{3}, \cdots, E_{m}$, we may assume that $\gamma_{123} \neq 0$. Thus we may introduce the new units

$$
E_{1}^{\prime}=E_{1}, \quad E_{2}^{\prime}=E_{2}, \quad E_{3}^{\prime}=\sum_{k=3}^{m} \gamma_{12 k} E_{k}, \quad E_{i}^{\prime}=E_{i}+\rho_{i} E_{3}^{\prime} \quad(i \geqq 4) .
$$

Then ${E_{3}^{\prime}}^{2}$ is the constant $\Sigma \gamma_{1 k}^{2} c_{k}$, not zero, which we shall designate by $c_{3}^{\prime}$. Taking $\rho_{i}=-c_{i} \gamma_{12 i} / c_{3}^{\prime}$, we find that

$$
E_{3}^{\prime} E_{i}^{\prime}+E_{i}^{\prime} E_{3}^{\prime}=0, \quad E_{j}^{\prime} E_{3}^{\prime}+E_{3}^{\prime} E_{j}^{\prime}=0, \quad E_{j}^{\prime} E_{i}^{\prime}+E_{i}^{\prime} E_{j}^{\prime}=0 \quad(i \geqq 4, j=1,2) .
$$

These relations obviously remain true when any $E_{i}^{\prime}(i \geqq 4)$ is replaced by a linear homogeneous function $E_{i}^{\prime \prime}$ of $E_{t}^{\prime}, \cdots, E_{m}^{\prime}$. By the argument leading to

*For the field of all real members we may take $c_{k}=-1$. Then $\sigma$ vanishes only if each $x_{i}=0$. Thus every element $X+0$ has an inverse $\left(2 x_{0}-X\right) / \sigma=X_{1}$. If multiplication is associative, $X Y=Z$ has the solution $Y=X_{1} Z$. 
(4), we can choose linearly independent functions $E_{t}^{\prime \prime}, \cdots, E_{m}^{\prime \prime}$ such that

$$
E_{k}^{\prime \prime 2}=c_{k}^{\prime \prime}, \quad E_{k}^{\prime \prime} E_{l}^{\prime \prime}+E_{l}^{\prime \prime} E_{k}^{\prime \prime}=0 \quad(k, l=4, \ldots, m ; l+k) .
$$

Hence, dropping accents, we obtain a set of independent units satisfying relations (4) and also

$$
E_{1} E_{2}=\gamma_{12}+\gamma_{121} E_{1}+\gamma_{122} E_{2}+E_{3}, \quad E_{i} E_{j}=\gamma_{i j}+\sum_{k=1}^{m} \gamma_{i j k} E_{k}
$$

We now add the hypothesis that multiplication is associative. Thus

$$
\begin{gathered}
E_{i}\left(E_{i} E_{j}\right)=c_{i} E_{i}, \quad\left(E_{j} E_{i}\right) E_{i}=c_{i} E_{i j} \\
0=E_{i}\left(E_{i} E_{j}\right)+\left(E_{i} E_{j}\right) E_{i}=2 \gamma_{i j} E_{i}+2 c_{i} \gamma_{i j i}+\sum_{k \neq i} \gamma_{i j k}\left(E_{i} E_{k}+E_{k} E_{i}\right) .
\end{gathered}
$$

Hence $\gamma_{i j}=0, \gamma_{i j i}=0$. By (4), $\gamma_{i j k}=-\gamma_{j i k}$. Hence $\gamma_{i j i}=0$. Thus (7) gives $E_{1} E_{2}=E_{3}$. Hence

$$
E_{3}^{2}=E_{1} E_{2} \cdot E_{1} E_{2}=-E_{1}^{2} E_{2}^{2}, \quad c_{3}=-c_{1} c_{2} .
$$

If $m \geqq 4, E_{1}\left(E_{2} E_{4}\right)=E_{3} E_{4}$. But

$\left(E_{3} E_{4}\right)^{2}=-c_{3} c_{4}, \quad\left(E_{1} E_{2} E_{4}\right)^{2}=-E_{1} E_{2} E_{1} E_{4} E_{2} E_{4}=E_{1}^{2}\left(E_{2} E_{4}\right)^{2}=-c_{1} c_{2} c_{4}$.

Thus $c_{3}=c_{1} c_{2}$, in contradiction with $c_{3}=-c_{1} c_{2}$. Hence $m=3$. Now

$$
E_{1} E_{3}=E_{1}\left(E_{1} E_{2}\right)=c_{1} E_{2}, \quad E_{3} E_{2}=\left(E_{1} E_{2}\right) E_{2}=c_{2} E_{1} \text {. }
$$

Hence, by (4), we have

$$
\begin{gathered}
E_{1}^{2}=c_{1}, \quad E_{2}^{2}=c_{2}, \quad E_{3}^{2}=-c_{1} \dot{c}_{2}, \quad E_{1} E_{2}=E_{3}=-E_{2} E_{1}, \\
E_{1} E_{3}=c_{1} E_{2}=-E_{3} E_{1}, \quad E_{3} E_{2}=c_{2} E_{1}=-E_{2} E_{3} .
\end{gathered}
$$

For this algebra we readily determine the conditions on $c_{1}$ and $c_{2}$ under which right and left hand division is always possible and unique. The general element may be written in the form $R+S E_{2}$, where $R=r+\rho E_{1}$ and $S=s+\sigma E_{1}$ belong to the field $F\left(E_{1}\right)$. Set $\bar{R}=r-\rho E_{1}$. Then $E_{2} R=\bar{R} E_{2}$. Hence

$$
\left(X+Y E_{2}\right)\left(R+S E_{2}\right)=X R+Y \bar{S} c_{2}+(X S+Y \bar{R}) E_{2} .
$$

For $R$ and $S$ not both zero, we require that this product shall equal an arbitrarily assigned element of the algebra. A necessary and sufficient condition is that

$$
\Delta=\left|\begin{array}{cc}
R & \bar{S} c_{2} \\
S & \bar{R}
\end{array}\right|=R \bar{R}-c_{2} S \bar{S}
$$

shall vanish only when $R=S=0$. For $S=0$, the condition requires that Trans. Am. Math. Soc. 6 
$r^{2}-\rho^{2} c_{1}$ shall vanish only for $r=\rho=0$, namely, that $c_{1}$ be a not-square. For $S \neq 0, R / S$ is an element $T$ of $F\left(E_{1}\right)$, and the condition requires that $c_{2}$ shall not be of the form $T \bar{T}$. Similarly,

$$
\left(R+S E_{2}\right)\left(X+Y E_{2}\right)=A+B E_{2}, \quad A=R X+S \bar{Y} c_{2}, \quad \bar{B}=\bar{S} X+\bar{R} \bar{Y},
$$

the determinant of the coefficients of $X$ and $\bar{Y}$ being $\Delta$. Hence right and left hand division is always possible and unique in algebra (8) if and only if $c_{1}$ is a notsquare in the field $F$ and $c_{2}$ is not expressible in the form $x^{2}-c_{1} y^{2}, x$ and $y$ in $F$.

We have now determined the linear associative algebras with coördinates in a field $F$ such that every element of the algebra satisfies a quadratic equation in $F$ and such that right and left hand division is always possible and unique. The algebra is either $F$ itself, a field $F\left(E_{1}\right)$ quadratic with respect to $F$, or one of type (8).

If $F$ is the field of all real numbers, $c_{1}$ and $c_{2}$ may be taken to be -1 and (8) is then the quaternion system. Since a real polynomial in $\rho$ is a product of real linear and quadratic factors, every element of an algebra satisfies a real quadratic equation. Hence the linear associative algebras in which division is unique and in which the coördinates range over the real numbers are the real, the complex and the quaternion number systems. This result is due to Frobenius* and C. S. PeIRCE. $\dagger$

7. Consider algebra (4) for $m=3$. Employing the notations (5) and $\left(7_{2}\right)$, we find that the minor of the first element of the first row of $\Delta(X)$ is

$$
M_{0}=\left|\begin{array}{rrr}
x_{0}+x_{2} \gamma_{211}+x_{3} \gamma_{311} & x_{1} \gamma_{121}+x_{3} \gamma_{321} & x_{1} \gamma_{131}+x_{2} \gamma_{231} \\
x_{2} \gamma_{212}+x_{3} \gamma_{312} & x_{0}+x_{1} \gamma_{122}+x_{3} \gamma_{322} & x_{1} \gamma_{132}+x_{2} \gamma_{232} \\
x_{2} \gamma_{213}+x_{3} \gamma_{313} & x_{1} \gamma_{123}+x_{3} \gamma_{323} & x_{0}+x_{1} \gamma_{133}+x_{2} \gamma_{233}
\end{array}\right| .
$$

From $M_{0}=Q x_{0}$, we find by inspection that

$$
Q=x_{0}^{2}+x_{0} \sum_{i, j} x_{i} \gamma_{i j}+\sum_{i, j} x_{i} x_{j}\left|\begin{array}{cc}
\gamma_{i j j} & \gamma_{j k j} \\
\gamma_{i j k} & \gamma_{j k k}
\end{array}\right|+\sum_{i} x_{i}^{2}\left|\begin{array}{cc}
\gamma_{i j j} & \gamma_{i k j} \\
\gamma_{i j k} & \gamma_{i k k}
\end{array}\right|,
$$

where $i, j, k$ form a permutation of $1,2,3$, while in the final sum $j<k$.

We discuss the algebras for which $Q \equiv \sigma$. By the coefficients of $x_{0}$,

$$
\gamma_{133}=-\gamma_{122}, \quad \gamma_{232}=-\gamma_{131}, \quad \gamma_{233}=\gamma_{121} .
$$

Then the conditions from the terms quadratic in $x_{1}, x_{2}, x_{3}$ become

$$
\begin{aligned}
\gamma_{122} \gamma_{121}+\gamma_{131} \gamma_{123} & =0, & \gamma_{131} \gamma_{122}-\gamma_{132} \gamma_{121} & =0, & \gamma_{121} \gamma_{131}+\gamma_{231} \gamma_{122} & =0, \\
\gamma_{122}^{2}+\gamma_{123} \gamma_{132} & =c_{1}, & \gamma_{121}-\gamma_{123} \gamma_{231} & =c_{2}, & \gamma_{131}^{2}+\gamma_{132} \gamma_{231} & =c_{3} .
\end{aligned}
$$

* Journal für reine und angewandte Mathematik, vol. 84 (1878), p. 59. $\dagger$ American Journal of Mathematics, vol. 4 (1881), p. 225. 
Let each $c_{i} \neq 0$. In the second and third equations, the determinant of the coefficients of $\gamma_{121}, \gamma_{122}$ is $-c_{3}$. Hence $\gamma_{121}=\gamma_{122}=0$. By the first equation, $\gamma_{131}=0$. Then

$$
\gamma_{132}=c_{1} / \gamma, \quad \gamma_{231}=-c_{2} / \gamma, \quad c_{1} c_{2}=-c_{3} \gamma^{2}, \quad \gamma \equiv \gamma_{123} .
$$

Replacing $E_{3}$ by $\gamma^{-1} E_{3}$, we have

$$
\begin{gathered}
E_{1}^{2}=c_{1}, \quad E_{2}^{2}=c_{2}, \quad E_{3}^{2}=-c_{1} c_{2}, \quad E_{1} E_{2}=E_{3}+\delta_{12}, \\
E_{1} E_{3}=c_{1} E_{2}+\delta_{13}, \quad E_{2} E_{3}=-c_{2} E_{1}+\delta_{23} .
\end{gathered}
$$

If the associative law holds, each $\delta_{i j}=0$ and the algebra becomes (8).

We may specialize the $\delta_{i j}$ by applying to the $E_{i}$ the transformation $(I+Z)^{-1}$ $(I-Z)$, an automorph of $q=c_{1} v_{1}^{2}+c_{2} v_{2}^{2}+c_{3} v_{3}^{2}$, where $I$ is the unit ma rix, $Z=M^{-1} Y, M$ being the matrix of $q$ and $Y$ being any skew symmetric matix. Here $c_{3}=-c_{1} c_{2}$. We find that $c_{1} E_{2} E_{3},-c_{2} E_{1} E_{3}, c_{3} E_{1} E_{2}$ are transformed cogrediently with $E_{1}, E_{2}, E_{3}$; likewise, $c_{1} \delta_{23},-c_{2} \delta_{13}, c_{3} \delta_{12}$. In particular, $c_{1} \delta_{23}^{2}+c_{2} \delta_{13}^{2}+c_{3} \delta_{12}^{2}$ is an absolute invariant.

C'ommutative linear algebras in the field of real numbers, with division always possible and unique.

8. The association law is not assumed. Let

$$
X_{m}+a_{1} X_{m-1}+\cdots+a_{m}=0
$$

be the equation of lowest degree with real coefficients satisfied by a given element $X$ of the algebra (cf. $\S 2$ ). If $X$ is not in the field of reals, we have $m>1$. Then $m$ must be even. For, if $m$ were odd, then

$$
\begin{aligned}
\rho^{m}+a_{1} \rho^{m-1}+\cdots+a_{m} & =(\rho+r)\left(\rho^{m-1}+b_{1} \rho^{m-2}+\cdots+b_{m-1}\right), \\
0=X_{m}+a_{1} X_{m-1}+\cdots+a_{m} & =(X+r)\left(X_{m-1}+\cdots+b_{m-1}\right),
\end{aligned}
$$

whereas each factor is of degree $<m$ and hence not zero. Iet $n$ be the number of units. If $n=2$, the algebra is the complex number system. Henceforth, let $n>2$. Since algebra (4) is not commutative, not every element satisfies a quadratic equation. Hence three of our units may be taken to be $1, e_{1}$, $e_{2}=e_{1}^{2}$, where $e_{1}$ does not satisfy a cubic equation. Thus $e_{1} e_{2}$ is linearly independent of $e_{1}$ and $e_{2}$. Hence we may take as the fourth unit $e_{3}=e_{1} e_{2}$.

Suppose that $e_{2}+a+b e_{1}+c e_{2}+d e_{3}=0$. In the field of reals let

Then

$$
x^{4}+a+b x+c x^{2}+d x^{3}=\left(x^{2}+e x+f\right)\left(x^{2}+g x+h\right) .
$$

Thus

$$
a=f h, \quad f=e h+f g, \quad c=f+h+e g, \quad d=e+g .
$$

$$
0=e_{2}^{2}+a+b e_{1}+c e_{2}+d e_{3}=\left(e_{2}+e e_{1}+f\right)\left(e_{2}+g e_{1}+h\right) \text {, }
$$


whereas neither factor is zero. Hence $n \geqq 5$ and we may take $e_{4}=e_{2}^{2}$ as the fifth unit.

For $n=5$, the general element $X$ satisfies an equation of degree $m$, where $m$ is even and $2<m \leqq 5$; hence $m=4$. For $n \geqq 6$, we make the assumption that $m=4$. Thus

$$
X \cdot X^{3}=A+B X+C X^{2}+D X^{3} .
$$

In particular, $e_{1} e_{3}=A+\cdots+D e_{3}$. Set $E_{1}=e_{1}+k, E_{2}=E_{1}^{2}, \cdots$. Then

$$
E_{1} E_{3}=\alpha+\beta E_{1}+\gamma E_{2}+(D+4 k) E_{3} .
$$

Hence we may set $D=0$ and

$$
e_{1} e_{3}=b e_{2}+c e_{1}+d \text {. }
$$

Then $t^{4}=b t^{2}+c t+d$ has no real root. For, if so,

$$
\left(e_{1}-t\right)\left[e_{3}+t e_{2}+\left(t^{2}-b\right) e_{1}+t^{3}-t b-c\right]=0 \text {. }
$$

Lemma. If $e$ is linearly independent of $1, e_{1}, e_{2}, e_{3}$, then $e_{1} e$ is linearly independent of $1, e_{1}, e_{2}, e_{3}, e$.

For, if $e_{1} e=\alpha e+\beta e_{3}+\gamma e_{2}+\delta e_{1}+\epsilon$, then

vanishes when

$$
\left(e_{1}-\alpha\right)\left(e+y e_{3}+z e_{2}+w e_{1}+\lambda\right)
$$

$$
\begin{gathered}
z=\alpha y-\beta, \quad w=\left(\alpha^{2}-b\right) y-\alpha \beta-\gamma, \quad \lambda=\left(\alpha^{3}-\alpha b-c\right) y-\alpha^{2} \beta-\alpha \gamma-\delta, \\
\left(\alpha^{4}-\alpha^{2} b-\alpha c-d\right) y=\alpha^{3} \beta+\alpha^{2} \gamma+\alpha \delta+\epsilon .
\end{gathered}
$$

Since $\alpha$ is real, the coefficient of $y$ is not zero.

Taking $e=e_{4}$, we see that $n>5$ and that the sixth unit may be chosen to be $e_{5}=e_{1} e_{4}$.

Let the algebra have only 6 units, $e_{0}=1, e_{1}, \cdots, e_{5}$. Then

(11) $e_{1}^{2}=e_{2}, \quad e_{1} e_{2}=e_{3}, \quad e_{2}^{2}=e_{4}, \quad e_{1} e_{3}=b e_{2}+c e_{1}+d, \quad e_{1} e_{4}=e_{5}$,

(12) $e_{1} e_{5}=\Sigma m_{i} e_{i}, \quad e_{2} e_{3}=\Sigma f_{i} e_{i}, \quad e_{2} e_{4}=\Sigma g_{i} e_{i}, \quad e_{2} e_{5}=\Sigma h_{i} e_{i}$.

Applying the Lemma for $e=x e_{4}+y e_{5}$, where $x$ and $y$ are not both zero, we see that $x e_{5}+y\left(m_{4} e_{4}+m_{5} e_{5}\right)$ is not a multiple of $x e_{4}+y e_{5}$. Hence

$$
\left|\begin{array}{cc}
y m_{4} & x+y m_{5} \\
x & y
\end{array}\right| \neq 0, \quad m_{5}^{2}+4 m_{4}<0 .
$$

Thus $m_{4}<0$ and we may set $m_{4}=-1 / r^{2}$. For $E_{i}=r^{i} e_{i}$, $E_{2}=E_{1}^{2}, \quad E_{3}=E_{1} E_{2}, \quad E_{4}=E_{2}^{2}, \quad E_{5}=E_{1} E_{4}, \quad E_{1} E_{5}=\sum_{i<4} m_{i}^{\prime} E_{i}-E_{4}+r m_{5} E_{5}$.

Hence we may set $m_{4}=-1,0 \leqq m_{5}<2$ in the initial formulas. 
The characteristic determinant for $X=x_{0}+x_{1} e_{1}+x_{2} e_{2}$ is

Set

$$
\Delta=\left|\begin{array}{rrrrrr}
x_{0} & 0 & 0 & x_{1} d+x_{2} f_{0} & x_{2} g_{0} & x_{1} m_{0}+x_{2} h_{0} \\
x_{1} & x_{0} & 0 & x_{1} c+x_{2} f_{1} & x_{2} g_{1} & x_{1} m_{1}+x_{2} h_{1} \\
x_{2} & x_{1} & x_{0} & x_{1} b+x_{2} f_{2} & x_{2} g_{2} & x_{1} m_{2}+x_{2} h_{2} \\
0 & x_{2} & x_{1} & x_{0}+x_{2} f_{3} & x_{2} g_{3} & x_{1} m_{3}+x_{2} h_{3} \\
0 & 0 & x_{2} & x_{2} f_{4} & x_{0}+x_{2} g_{4} & -x_{1}+x_{2} h_{4} \\
0 & 0 & 0 & x_{2} f_{5} & x_{1}+x_{2} g_{5} & x_{0}+x_{1} m_{5}+x_{2} h_{5}
\end{array}\right| .
$$

$$
X^{3}-B-C X-D X^{2}=\Sigma c_{i} e_{i}
$$

Then, by (10) and $\S 4$, the co-factor $F_{i}$ of the $i$ th element of the first row of $\Delta$ equals $Q c_{i}$, where $Q=\Delta / A$. Now

$$
c_{5}=x_{1} x_{2}^{2}\left(1+2 f_{5}\right)+x_{2}^{3} g_{5}, \quad c_{4}=x_{1}^{2} x_{2}+x_{2}^{2} l, \quad l \equiv 3 x_{0}+g_{4} x_{2}-D .
$$

Thus $F_{6}$ has the factor $x_{2}^{2}$. But

$$
F_{5}=x_{1}^{3} x_{2}\left\{x_{0}\left(1+f_{5}\right)-x_{1} f_{4}\right\}+x_{2}^{2}() \text {. }
$$

Hence $f_{4}=0, f_{5}=-1, c_{5}=x_{2}^{2}\left(g_{5} x_{2}-x_{1}\right)$. Then $F_{5}$ is divisible by $c_{3}$ if and only if

$g_{3}=g_{5}^{3}+2 g_{5}\left(g_{4}-f_{3}\right), \quad g_{1}=g_{2} g_{5}-2 f_{1} g_{5}+\left(2 f_{2}-g_{3}-2 c\right) g_{5}^{2}+\left(g_{4}+2 b-2 f_{3}\right) g_{5}^{3}$, the quotient being

$$
\begin{gathered}
Q=-x_{0}^{2}-g_{5} x_{0} x_{1}+x_{0} x_{2}\left(f_{3}-2 g_{4}-g_{5}^{2}\right)+x_{1}^{2}\left(b+g_{4}-f_{3}\right)+x_{1} x_{2} Y+x_{2}^{2} Z, \\
Y \equiv f_{2}-g_{3}-c+\left(g_{4}+2 b-2 f_{3}\right) g_{5}, \\
Z \equiv g_{2}-f_{1}+\left(2 f_{2}-g_{3}-2 c\right) g_{5}+\left(g_{4}+2 b-2 f_{3}\right) g_{5}^{2} .
\end{gathered}
$$

The conditions for $F_{4}=Q c_{4}$ reduce to $l=-x_{0}-(b+1) x_{2}$ and $^{*}$

$$
\begin{gathered}
m_{5}=g_{5}, \quad f_{3}=g_{4}+b+1, \quad h_{5}=g_{5}^{2}-b-1, \quad h_{4}=f_{2}-c-g_{5}, \quad m_{3}=g_{3}, \\
h_{3}=2 f_{1}-g_{2}-2 f_{2} g_{5}+2 c g_{5}+g_{3} g_{5}+2 g_{5}^{2}+2 g_{4}(b+1), \\
m_{2}=f_{1}-f_{2} g_{5}+c g_{5}+g_{5}^{2}+g_{4}(b+1), \\
h_{2}=m_{1}+g_{5} m_{2}+b g_{3}, \quad h_{1}=Z(b+1)-h_{5} f_{1} .
\end{gathered}
$$

Giving notations to $e_{3}^{2}, \cdots, e_{4} e_{5}$, we set

(13) $X=\Sigma x_{i} e_{i}, \quad X^{2}=\Sigma L_{i} e_{i}, \quad X^{3}=\Sigma G_{i} e_{i}, \quad X \cdot X^{3}=\Sigma M_{i} e_{i}(i=0,1, \ldots, 5)$.

* These and the earlier relations are together equivalent to those obtained by requiring that $e_{2}+x e_{1}$ shall satisfy an equation (10). 
Then, by (10),

$$
M_{i}=B x_{i}+C L_{i}+D G_{i}
$$$$
(i=1, \ldots, 5) \text {. }
$$

First, set $x_{0}=x_{4}=x_{5}=0$. Eliminating $B$, and then $C, D$ we have

$$
\Delta_{i j}=\left|\begin{array}{ccc}
M_{i} x_{j}-M_{j} x_{i} & L_{i} x_{j}-L_{j} x_{i} & G_{i} x_{j}-G_{j} x_{i} \\
M_{4} & L_{4} & G_{4} \\
M_{5} & L_{5} & G_{5}
\end{array}\right|=0 \quad(i, j=1,2,3) .
$$

The terms with the factor $x_{1}^{6}$ in $\Delta_{23}$ and $\Delta_{13}$ were obtained and the resulting conditions on the constants derived. Also $L_{4} M_{5}-L_{5} M_{4}$ must be divisible by $L_{4} G_{5}-L_{5} G_{4}$, the quotient being $D$. Finally, $M_{5}-D G_{5}$ must be divisible by $L_{5}$, the quotient being $C$. Similarly, for $x_{0}=x_{2}=x_{3}=0$, the analogous determinants $\Delta_{15}$ and $\Delta_{45}$ were examined; by the terms of highest degree in $x_{1}$, we get

$$
g_{4}=3+3 b-4 g_{5}^{2}, \quad f_{2}=c+4 g_{5}-2 g_{5}^{3} .
$$

For $x_{0}=x_{5}=0$, the terms in $x_{1}^{2} x_{4}^{2}, x_{1} x_{2}^{2} x_{4}, x_{1} x_{2} x_{3} x_{4}$ of $M_{5}-D G_{5}-C L_{5}=0$ yield

$$
m_{2}=g_{2}+4+8 b+4 b^{2}-8 b g_{5}^{2}-4 g_{5}^{2}+3 g_{5}^{4} \text {. }
$$

Hence, writing $g=g_{5}$,

$$
\begin{gathered}
Q=-x_{0}^{2}-g x_{0} x_{1}+\left(3 g^{2}-2-2 b\right) x_{0} x_{2}-x_{1}^{2}+\left(g+g^{3}-b g\right) x_{1} x_{2} \\
+\left(2 g^{2}-2 g^{4}+3 b g^{2}-b^{2}-2 b-1\right) x_{2}^{2}, \\
-4 Q=\left[2 x_{0}+g x_{1}+\left(2+2 b-3 g^{2}\right) x_{2}\right]^{2}+\left(4-g^{2}\right)\left(x_{1}-g x_{2}\right)^{2}
\end{gathered}
$$

the additional term in $x_{2}^{2}$ having the coefficient zero. Hence $Q$ and therefore $\Delta$ vanishes for values of the $x_{i}$ not all zero, so that division is not always uniquely possible.

Theorem. If division is always possible and unique in a commutative linear algebra with coördinates ranging over all real numbers, the number of units is at least six. If there are only six units, the characteristic sextic is the equation of lowest degree satisfied by the general element of the algebra.

Commutative linear algebras with six units in which every element is a root of a quartic equation.

9. Consider a commutative algebra of type (11)-(12), in which every element satisfies a quartic equation, and $m_{4}=-1$, as before. Replacing $e_{1}$ by $e_{1}+k$, we may take $c=0$. To s mplify the formulas and computation $\operatorname{set} b=-1$, 
$g_{2}=g_{5}=m_{1}=0$. The only such algebras may be shown to be those satisfying (11) and

$$
\begin{gathered}
e_{1} e_{5}=m-e_{4}, \quad e_{2} e_{3}=f-e_{5}, \quad e_{2} e_{4}=g, \quad e_{2} e_{5}=h, \\
e_{3}^{2}=\alpha-e_{4}, \quad e_{3} e_{4}=\delta, \quad e_{3} e_{5}=E, \quad e_{4}^{2}=\beta, \quad e_{4} e_{5}=K, \quad e_{5}^{2}=\gamma .
\end{gathered}
$$

We shall prove that in a commutative algebra defined by (11) and (15), every element $\Sigma x_{i} e_{i}$ satisfies a quartic equation. It suffices to treat the elements with $x_{0}=0$. Then, in (13),

$$
\begin{array}{r}
L_{0}=2 d x_{1} x_{3}+2 m x_{1} x_{5}+2 f x_{2} x_{3}+2 g x_{2} x_{4}+2 h x_{2} x_{5}+\alpha x_{3}^{2}+2 \delta x_{3} x_{4}+2 E x_{3} x_{5} \\
+\beta x_{4}^{2}+2 K x_{4} x_{5}+\gamma x_{5}^{2}, \\
L_{1}=0, \quad L_{2}=x_{1}^{2}-2 x_{1} x_{3}, \quad L_{3}=2 x_{1} x_{2}, \\
L_{4}=-2 x_{1} x_{5}+x_{2}^{2}-x_{3}^{2}, \quad L_{5}=2 x_{1} x_{4}-2 x_{2} x_{3}, \\
\begin{array}{r}
G_{0}=f\left(L_{2} x_{3}+L_{3} x_{2}\right)+g\left(L_{2} x_{4}+L_{4} x_{2}\right)+h\left(L_{2} x_{5}+L_{5} x_{2}\right)+d L_{3} x_{1}+\alpha L_{3} x_{3} \\
+\delta\left(L_{3} x_{4}+L_{4} x_{3}\right)+E\left(L_{3} x_{5}+L_{5} x_{3}\right)+\beta L_{4} x_{4}
\end{array} \\
+K\left(L_{4} x_{5}+L_{5} x_{4}\right)+m L_{5} x_{1}+x L_{5} x_{5}, \\
G_{4}=L_{0} x_{4}+L_{2} x_{2}-L_{3} x_{3}-L_{5} x_{1}, \quad G_{5}=L_{0} x_{5}-L_{2} x_{3}-L_{3} x_{2}+L_{4} x_{1}, \\
M_{1}=G_{0} x_{1}, \quad M_{2}=G_{0} x_{2}+G_{1} x_{1}-G_{1} x_{3}-G_{3} x_{1}, \quad M_{3}=G_{0} x_{3}+G_{1} x_{2}+G_{2} x_{1}, \\
M_{4}=G_{0} x_{4}-G_{1} x_{5}+G_{2} x_{2}-G_{3} x_{3}-G_{5} x_{1},
\end{array}
$$$$
M_{5}=G_{0} x_{5}+G_{1} x_{4}-G_{2} x_{3}-G_{3} x_{2}+G_{4} x_{1} .
$$

We obtain $M_{0}$ from $G_{0}$ by replacing $L_{i}$ by $G_{i}$ and adding $d G_{1} x_{3}+m G_{1} x_{5}$.

We may now show that $X$ satisfies a quartic equation (10) with $D=0$. Consider the conditions (14). For $i=1$, we get $M_{1}=B x_{1}$, whence $B=G_{0}$. Then for $i=3$,

$$
G_{1} x_{2}+G_{2} x_{1}=2 x_{1} x_{2} C, \quad 2 x_{1} x_{2}\left(L_{0}-x_{1}^{2}-C\right)=0, \quad C=L_{0}-x_{1}^{2} .
$$

For these values of $B, C, D$, conditions (14), with $i=2,4,5$, are seen at once to become identities when the $G$ 's are expressed in terms of the $L$ 's and $L_{i}$ in the one term $L_{0} L_{i}$ is expressed in terms of the $x$ 's. Finally, there remains only the condition $M_{0}=A+C L_{0}$. Hence $X=x_{1} e_{1}+\cdots+x_{5} e_{5}$ is a root of

$$
X \cdot X^{3}=M_{0}-L_{0}\left(L_{0}-x_{1}^{2}\right)+G_{0} X+\left(L_{\theta}-x_{1}^{2}\right) X^{2} .
$$


In particular, when $d, m, \cdots, \gamma$ are zero, we have

$$
\begin{array}{r}
e_{1}^{2}=e_{2}, \quad e_{1} e_{2}=e_{3}, \quad e_{1} e_{3}=-e_{2}, \quad e_{1} e_{4}=e_{5}, \quad e_{1} e_{5}=e_{3}^{2}=-e_{4}, e_{2}^{2}=e_{4}, \\
e_{2} e_{3}=-e_{5}, \quad e_{2} e_{4}=e_{2} e_{5}=e_{3} e_{4}=e_{3} e_{5}=e_{4}^{2}=e_{4} e_{5}=e_{5}^{2}=0 .
\end{array}
$$

In a commutative algebra of this type, $x_{1} e_{1}+\cdots+x_{5} e_{5}$ is a root of

$$
X \cdot X^{3}+x_{1}^{2} X^{2}=0,
$$

but not of a cubic or quadratic equation. The general element $x=X+x_{0}$ is therefore a root of

$$
x \cdot x^{3}-4 x_{0} x^{3}+\left(x_{1}^{2}+6 x_{0}^{2}\right) x^{2}-\left(2 x_{0} x_{1}^{2}+4 x_{0}^{3}\right) x+x_{0}^{2} x_{1}^{2}+x_{0}^{4}=0 .
$$

Uniqueness of division in Cayley's algebras with eight units.

10. CAYley * gave, as a direct generalization of quaternions, a linear algebra with eight units in which the modulus of a product equals the product of the moduli of the factors, each modulus being a sum of eight squares. By changing the sign of the final unit we obtain one of the two algebras obtained by CAYLEY $\dagger$ in his complete enumeration of certain types of algebras which possess this property of eight squares. Either of these two algebras may be obtained by changing the signs of $e_{2}, \cdots, e_{7}$ in the other. The algebra are distinguished by the sign of $\epsilon_{7}= \pm 1$; that with $\epsilon_{7}=+1$ has the units $1, e_{1}, \cdots, e_{7}$, where

$$
\begin{array}{r}
e_{i}^{2}=-1, \quad e_{i} e_{j}=-e_{j} e_{i} \quad(i, j=1, \cdots, 7 ; i \neq j), \\
e_{1} e_{2}=e_{3}, \quad e_{1} e_{4}=e_{5}, \quad e_{1} e_{6}=e_{7}, \quad e_{2} e_{5}=e_{7}, \quad e_{3} e_{4}=e_{7}, \quad e_{3} e_{5}=e_{6}, \quad e_{4} e_{2}=e_{6},
\end{array}
$$

with fourteen equations obtained by permuting the subscripts cyclically

Since the modulus of any element is the sum of the squares of its coördinates, any element except zero has an inverse, the coördinates being assumed real. Since the associative law does not hold, the existence of an inverse does not imply uniqueness of division. To prove the latter, we note that every element may be expressed as a linear function of $e_{2}, e_{4}, e_{6}$, with coefficients linear in $e_{1}$. For $B=r+s e_{1}$, set $\bar{B}=r-s e_{1}$. Let also $\bar{C}$ be linear in $e_{1}$. Then

$$
\begin{array}{r}
e_{j} B=\bar{B} e_{j}, \quad\left(B e_{j}\right)\left(C e_{j}\right)=(B \bar{C}) e_{j}^{2}, \quad\left(B e_{j}\right)\left(C e_{k}\right)=(\bar{B} \bar{C})\left(e_{j} e_{k}\right) . \\
(j, k=2,4,6 ; j \neq k) .
\end{array}
$$

Hence if the coefficients are linear in $e_{1}$, we have

$$
\begin{gathered}
\left(A+B e_{2}+C e_{4}+D e_{6}\right)\left(\alpha+\beta e_{2}+\gamma e_{4}+\delta e_{6}\right)=P+Q e_{2}+R e_{4}+S e_{6}, \\
P=A \alpha-B \bar{\beta}-C \bar{\gamma}-D \bar{\delta}, \quad Q=A \beta+B \bar{\alpha}-\bar{C} \bar{\delta}+\bar{D} \bar{\gamma}, \\
R=A \gamma+\bar{B} \bar{\delta}+C \bar{\alpha}-\bar{D} \bar{\beta}, \quad S=A \delta-\bar{B} \bar{\gamma}+\bar{C} \bar{\beta}+D \bar{\alpha} .
\end{gathered}
$$

*Philosophical Magazine, London, (3), vol. 26, (1845), p. 210.

† American Journal of Mathematics, vol. 4 (1881), pp. 293-296. 
By multiplication and addition we derive

$$
\begin{aligned}
\sigma A & =\bar{\alpha} P+\bar{\beta} Q+\bar{\gamma} R+\bar{\delta} S, & \sigma B & =-\beta P+\alpha Q+\delta \bar{R}-\bar{\gamma} \bar{S}, \\
\sigma C & =-\gamma P-\bar{\delta}+\alpha R+\bar{\beta} \bar{S}, & \sigma D & =-\delta P+\bar{\gamma} \bar{Q}-\bar{\beta} \bar{R}+\alpha S, \\
s \alpha & =\bar{A} P+B \bar{Q}+C \bar{R}+D \bar{S}, & s \beta & =-B \bar{P}+\bar{A} Q-\bar{D} \bar{R}+\bar{C} \bar{S}, \\
s \gamma & =-C \bar{P}+\bar{D} \bar{Q}+\bar{A} R-\bar{B} \bar{S}, & s \delta & =-D \bar{P}-\bar{C} \bar{Q}+\bar{B} \bar{R}+\bar{A} S, \\
\sigma & =\alpha \bar{\alpha}+\beta \bar{\beta}+\gamma \bar{\gamma}+\delta \bar{\delta}, & s & =A \bar{A}+B \bar{B}+C \bar{C}+D \bar{D} .
\end{aligned}
$$

Hence if $G$ and $H$ are any given elements, $G \neq 0$, there is an unique solution $X$ or $Y$ of $X G=H$ or $G Y=H$. Right and left hand division except by zero is always possible and unique in these Cayley algebras. 